\title{
Non-Alcoholic Fatty Liver Disease and the Left Ventricle Mass Index in Obese Children
}

Beray Selver ${ }^{1}$, Enver Simsek ${ }^{2 *}$, Ugur Kocabas ${ }^{3}$ and Yildiz Dallar ${ }^{1}$

${ }^{1}$ Department of Pediatrics, Ankara Research and Training Hospital, Ankara, Turkey

${ }^{2}$ Department of Pediatrics, Division of Endocrinology, Eskisehir Osmangazi University, School of Medicine, Eskisehir, Turkey

${ }^{3}$ Department of Cardiology, Ankara Research and Training Hospital, Ankara, Turkey

\begin{abstract}
Objective: To investigate Nonalcoholic Fatty Liver Disease (NAFLD), the Left Ventricle Mass Index (LVMI), and the relationship between NAFLD and LVMI in obese children.

Material and methods: Systolic (SBP) and Diastolic (DBP) Blood Pressure and waist and hip circumferences were measured. Fasting blood glucose and insulin concentrations, total cholesterol, and Triglycerides (TG) were assayed. The diagnosis of NAFLD was based on sonographic evidence of a fatty liver. The Left Ventricle Mass (LVM) was calculated from two-dimensionally guided M-mode echocardiographic measurements of the left ventricle. LVMI was calculated as LVM $(\mathrm{g}) /$ height $(\mathrm{m})^{2.7}$ and Left Ventricular Hypertrophy $(\mathrm{LVH})$ was defined as $\mathrm{LVMI} \geq 95^{\text {th }}$ percentile for age and gender
\end{abstract}

Results: Forty-three obese children with NAFLD, 55 obese children without NAFLD, and 48 non-obese controls were studied. Fasting insulin, homeostasis model assessment-estimated insulin resistance (HOMA-IR) index, TG, and total cholesterol levels in the obese children were significantly higher than in the controls (all $p<0.001)$; SBP and DBP in the obese children were also higher than in the controls. LVMI was higher in the obese children $(p<$ $0.001)$, although the mean LVMI did not differ significantly between obese children with and without NAFLD $(p>$ 0.05). The prevalence of LVH differed significantly between the obese groups and controls (all $p<0.001)$, while there was no significant difference between the obese subjects with and without NAFLD. LVH was present in 5 of 48 $(10.4 \%)$ control subjects, 25 of $79(31.6 \%)$ obese subjects with normotensive subjects, and 6 of $19(36.8 \%)$ obese subjects with hypertension. The prevalence of LVH differed significantly between the obese groups and controls (all $p<0.001$ ), whereas no significant difference was observed between obese subjects with or without hypertension $(p>0.05)$. In a multiple linear regression analysis, NAFLD, SBP, and DBP were not correlated with LVMI. The LVMI was closely related to the Body Mass Index-Standard Deviation Score (BMI-SDS), and Liver Longitudinal Dimension (LLD) percentile. The BMI-SDS was the only independent predictor of NAFLD and LVMI.

Conclusions: LVH and NAFLD are two important and independent covariates in obese children. Obese children with or without hypertension have significant LVH as compared with non-obese control subjects. NAFLD and casual blood pressure measurements are not predictors of LVMI in obese children.

Keywords: Children; Obesity; Left ventricle mass index; Nonalcoholic fatty liver disease

\section{Introduction}

The prevalence of childhood obesity is increasing, not only in developed countries, but also in some developing countries [1]. It is associated with several risk factors for later heart disease and other chronic diseases, including hyperlipidemia, hyperinsulinemia, hypertension, and early atherosclerosis [2]. Children who are overweight and obese are more likely to become overweight and obese adults [3].

Non-Alcoholic Fatty Liver Disease (NAFLD) describes a spectrum of liver damage, ranging from simple steatosis to steatohepatitis, advanced fibrosis, and cirrhosis in persons who have not consumed alcohol so as to cause liver damage, and in whom no other etiology for fatty liver is present. An increased prevalence of NAFLD has been observed along with a dramatic rise in obesity in children during the past three decades. Obesity and insulin resistance are hallmarks of NAFLD. The exact prevalence of NAFLD is unclear. Pooling data from studies performed primarily in tertiary medical centers, the reported prevalence of NAFLD in obese children ranges from 20 to $77 \%[1,4-6]$.

Left Ventricular Hypertrophy (LVH) has prognostic importance in adults and is generally considered to have a similar prognostic value in children. Numerous population-based and hypertensive cohort studies investigating the impact of adiposity on left ventricle structure have shown that $\mathrm{LVH}$ is more prevalent in obese individuals than in their lean counterparts $[7,8]$. No reported study has analyzed the relationship between NAFLD and the Left Ventricle Mass Index (LVMI) in obese children. Thus, this study investigated the risk factors for left ventricle hypertrophy and determined the relationship between NAFLD and the LVMI in obese children.

\section{Material and Methods}

This was a cross-sectional study that included 98 obese children and 48 age- and gender-matched non-obese children as a control group. Subjects were enrolled randomly from among the obese children admitted to the Pediatric Endocrinology Department of Ankara Research and Training Hospital. To compare variables that differed significantly between the obese children and controls, the obese children were subdivided into two groups according to the presence of NAFLD.

*Corresponding author: Prof. Dr. Enver Simsek, Department of Pediatrics, Division of Pediatric Endocrinology, Eskisehir Osmangazi University, School of Medicine, Eskisehir, Turkey, Tel: +9050549623402; E-mail: enversimsek06@hotmail.com

Received February 24, 2012; Accepted May 26, 2012; Published May 25, 2012

Citation: Selver B, Simsek E, Kocabas U, Dallar Y (2012) Non-Alcoholic Fatty Liver Disease and the Left Ventricle Mass Index in Obese Children. J Metabolic Synd 1:110. doi:10.4172/2167-0943.1000110

Copyright: (C) 2012 Selver B. This is an open-access article distributed under the terms of the Creative Commons Attribution License, which permits unrestricted use, distribution, and reproduction in any medium, provided the original author and source are credited. 
Inclusion criteria included a Body Mass Index (BMI) $\geq 95^{\text {th }}$ percentile for age and gender. Children were excluded if they had any condition known to influence body composition, insulin action, or insulin secretion (e.g., glucocorticoid therapy, hypothyroidism, Cushing's syndrome), or a history of medication use that could affect body mass or lipid profile. Children were also excluded if they had a history of medication use that causes liver inflammation, diabetes mellitus, or tested positive for hepatitis B or C.

At enrollment, the subjects all underwent physical examinations that included weight, standing height, BMI, and blood pressure measurements. Height was measured to the nearest $0.1 \mathrm{~cm}$ without shoes using a Harpenden stadiometer (Harpenden, Holtain, UK). Weight was measured to the nearest $0.1 \mathrm{~kg}$ on a standard beam scale with the subject dressed only in light underwear and without shoes. All measurements were repeated twice.

The weight status was recorded as the BMI, which was calculated as weight $(\mathrm{kg}) /$ height $^{2}(\mathrm{~m})$. Because the BMI changes rapidly with normal growth, and varies with age and gender, it was standardized for age and gender by converting to a " $\mathrm{z}$ score" [9]. Data were expressed as the Body Mass Index-Standard Deviation Score (BMI-SDS), which was calculated as [individual measurement-population mean]/population SD. Obesity was defined as a $\mathrm{BMI} \geq 95^{\text {th }}$ percentile according to reference curves for Turkish children [10]. The waist circumference was measured at the smallest point between the iliac crest and rib cage, and the hip circumference was measured at the largest width over the greater trochanter.

The resting Systolic (SBP) and Diastolic (DBP) Blood Pressures were measured twice in the right arm after a 10-min rest in a supine position by one investigator using a standard mercury sphygmomanometer and a validated protocol [11]. All subjects were considered hypertensive when the SBP or DBP was $\geq 95^{\text {th }}$ percentile for age, gender, and height according to a percentiles chart for Turkish children [12]. Patients with evidence of secondary hypertension and those already on antihypertensive medication were excluded. Secondary hypertension was excluded by history, physical examination, serum chemistry, urinalysis, renal ultrasound, and other tests as indicated. Obese subjects were divided into four groups (with or without NAFLD and with or without hypertension) to compare the frequency of LVMI and LVH.

\section{Biochemical analysis}

After an overnight fast, blood samples were taken for the analysis of serum glucose, plasma insulin, Total Cholesterol (TC), Triglycerides (TG), Alanine Aminotransferase (ALT), Aspartate Aminotransferase (AST), Hepatitis B surface Antigen ( $\mathrm{HBsAg}$ ), and anti-Hepatitis C Virus antibody (anti-HCV). Glucose was measured using the glucoseoxidase colorimetric method using an automated analyzer (Hitachi, Roche Diagnostics, Mannheim, Germany). Fasting insulin was analyzed with a radio-immunochemical method (Pharmacia \& Upjohn Diagnostics AB, Uppsala, Sweden). The detection limit was $0.5 \mu \mathrm{U} / \mathrm{mL}$, and the intra- and interassay coefficients of variation were 6.5 and $9.1 \%$, respectively. The homeostasis model assessment of insulin resistance (HOMA-IR) was used to evaluate insulin sensitivity [13]. The HOMAIR was calculated as HOMA-IR $=$ [fasting insulin $(\mu \mathrm{U} / \mathrm{mL}) \times$ fasting glucose $(\mathrm{mmol} / \mathrm{L})]$ / 22.5. A HOMA-IR > 3.16 was taken as a surrogate measure of insulin resistance [14].

The ALT, AST, fasting TC, and TG were measured using enzymatic colorimetric methods on an automated analyzer (Hitachi 904, Roche Diagnostics). Elevated liver enzymes were defined an ALT or AST $\geq 40$ units/L. Cut-off points $\geq 95^{\text {th }}$ percentile of healthy children were used to define dyslipidemia in accordance with international recommendations.
These cut-off points were $200 \mathrm{mg} / \mathrm{dL}(5.1 \mathrm{mmol} / \mathrm{L})$ for cholesterol and $150 \mathrm{mg} / \mathrm{dL}(1.7 \mathrm{mmol} / \mathrm{L})$ for triglycerides [15]. HBsAg and anti-HCV were measured using microparticle enzyme immunoassays (Abbott Laboratories, Abbott Park, IL, USA).

\section{Liver ultrasound and echocardiographic examination}

An ultrasound examination was performed to identify NAFLD and to measure the Liver Longitudinal Dimension (LLD). The procedure was carried out by an experienced radiologist who was unaware of both the study aims and the participants' biochemical profiles. An ultrasound device (GE LOGIQa 100 MP, GE Medical System, Milwaukie, WI, USA) with a $5-\mathrm{mHz}$ probe in younger children and a $3.5-\mathrm{mHz}$ probe in larger or markedly obese children was used. Fatty liver was diagnosed based on the ultrasonographic pattern and graded as absent, mild, moderate, and severe, according to the criteria of Needleman et al. [16]. In this study, the diagnosis of NAFLD was based on sonographic evidence of a fatty liver and negative test results for HBsAg and anti-HCV antibody. No participant had a history of liver disease, hypertension, or diabetes.

Echocardiograms were performed in all study subjects to evaluate LVH. All echocardiography was conducted by a single cardiologist. To determine the Left Ventricular Mass (LVM), two-dimensional echocardiography was performed in M-mode (General Electric Vivid 3, Norway) according to the guidelines of the American Society of Echocardiography [17]. All measurements were made at enddiastole, which was defined as the time of the maximum left ventricle dimension. Electronic calipers were used to measure the left ventricle internal diameter, interventricular septal thickness, and left ventricle posterior wall thickness from the M-mode images. The measurements were repeated over three consecutive cardiac cycles and averaged. The Devereux formula was used to estimate the LVM [18]. Because the LVM increases during growth and normal must be defined in the context of body size, the Left Ventricular Mass Index (LVMI) was calculated for all children as the LVM in grams divided by height in meters to the $2.7^{\text {th }}$ power $[19,20]$. Using pediatric criteria, Left Ventricle Hypertrophy (LVH) was defined as $L V M I \geq 95^{\text {th }}$ percentile for gender, which was $39.36 \mathrm{~g} / \mathrm{m}^{2.7}$ for boys and $36.88 \mathrm{~g} / \mathrm{m}^{2.7}$ for girls [21].

The study was conducted in accordance with the guidelines proposed in the Declaration of Helsinki and was approved by the Ethics Committee of Ankara Research and Training Hospital. Written informed consent was obtained from all participants over 12 years of age, and informed parental consent was obtained for all children regardless of age.

\section{Statistical Analysis}

Statistical analyses were performed using the Statistical Package for the Social Sciences (SPSS/PC+, ver. 13.0, Chicago, IL, USA). The following variables were included in the analysis: clinical data [Weight Standard Deviation Score (WSDS), Height Standard Deviation Score (HSDS), BMI-SDS, SBP, DBP, and pubertal status], biochemical parameters (fasting serum insulin and glucose concentrations, and HOMA-IR), LLD, NAFLD, LVM, and LVMI. The data are expressed as the mean \pm standard deviation (SD) or median (min-max) where appropriate. Test selection was based on evaluating the variables for a normal distribution using the Shapiro-Wilk test. If the variables were distributed normally, a Student's $t$-test was used; otherwise, the Mann-Whitney $U$-test was used. Categorical data were evaluated using Pearson's chi-squared or Fisher's exact test where applicable. Statistical correlations were calculated using Spearman's correlation. Multiple linear regression analysis was performed with LVMI and NAFLD as dependent variables, and age, BMI-SDS, HOMA-IR, AST, ALT, and LLD 
Citation: Selver B, Simsek E, Kocabas U, Dallar Y (2012) Non-Alcoholic Fatty Liver Disease and the Left Ventricle Mass Index in Obese Children. J Metabolic Synd 1:110. doi:10.4172/2167-0943.1000110

Page 3 of 7

\begin{tabular}{|c|c|c|c|}
\hline & Obese children $n=98(\%)$ & Non-obese controls $n=48(\%)$ & $\mathrm{p}$ \\
\hline Age (year) & $12.4 \pm 2.4$ & $11.6 \pm 2.2$ & $0.385^{\mathrm{a}}$ \\
\hline Boy/Girl & $41 / 57$ & $27 / 21$ & $0.101^{b}$ \\
\hline Pubertal/Prepubertal & $67 / 31$ & $31 / 17$ & $0.648^{b}$ \\
\hline BMI-SDS & $2.6(0.55-5.14)$ & $0.42(-1.71-1.94)$ & $<0.001^{c}$ \\
\hline Waist circumference $(\mathrm{WC})(\mathrm{cm})$ & $86(63-117)$ & $62(49-79)$ & $<0.001^{\mathrm{c}}$ \\
\hline Hip circumference $(\mathrm{HC})(\mathrm{cm})$ & $99.9 \pm 11.9$ & $78.8 \pm 9.1$ & $<0.001^{a}$ \\
\hline $\mathrm{WC} / \mathrm{HC}$ ratio & $0.88 \pm 0.087$ & $0.80 \pm 0.082$ & $<0.001^{a}$ \\
\hline SBP > 95th percentile & $19(19.4 \%)$ & none & $<0.001^{b}$ \\
\hline DBP > 95th percentile & $14(14.3 \%)$ & none & $0.005^{d}$ \\
\hline Glucose (mg/dl) & $82(53-225)$ & $84(67-98)$ & $0.154^{\circ}$ \\
\hline Insulin $(\mu \mathrm{U} / \mathrm{ml})$ & $17.8(2.3-113.6)$ & $9.2(3.4-22.7)$ & $<0.001^{c}$ \\
\hline HOMA-IR & $3.6(0.5-21.3)$ & $1.9(0.7-4.4)$ & $<0.001^{c}$ \\
\hline Triglyceride (mg/dl) & $124(38-432)$ & $82(33-207)$ & $<0.001^{\mathrm{c}}$ \\
\hline Total cholesterol (mg/dl) & $165 \pm 27$ & $141 \pm 24$ & $<0.001^{a}$ \\
\hline AST (U/L) & $23(11-96)$ & $24(15-40)$ & $0.742^{c}$ \\
\hline ALT (U/L) & $19(7-199)$ & $17(9-35)$ & $<0.001^{c}$ \\
\hline NAFLD & $43(43.9 \%)$ & $1(2.1 \%)$ & $<0.001^{\mathrm{b}}$ \\
\hline LVM (g) & $119.8(63.3-243.5)$ & $82.0(32.3-128.7)$ & $<0.001^{c}$ \\
\hline LVMI (g/m2.7) & $40.1(26.4-98.2)$ & $29.7(15.6-42.0)$ & $<0.001^{c}$ \\
\hline
\end{tabular}

SBP: Systolic Blood Pressure; DBP: Diastolic Blood Pressure; HOMA-IR: Homeostasis Model Assessment Of Insulin Resistance; AST: Aspartate Aminotransferase; ALT: Alanine Aminotransferase; NAFLD: Non-Alcoholic Fatty Liver Disease; LLD: Liver Longitudinal Dimension; LVM: Left Ventricle Mass; LVMI: Left Ventricle Mass Index. aStudent's t-test

'Pearson's chi-squared test

'Mann Whitney U test

'Fisher's exact test

Table 1: Clinical characteristics, laboratory data, LLD percentile, LVM, and LVMI in obese children and non-obese controls.

\begin{tabular}{|c|c|c|c|c|c|c|}
\hline & Obese children with NAFLD $(\mathrm{N}=43)$ & Obese children without NAFLD $(\mathrm{N}=55)$ & Control subjects $(\mathrm{N}=48)$ & p1 & $p 2$ & p3 \\
\hline BMI-SDS & $2.55(2.27-2.84)$ & $2.66(2.37-2.95)$ & $0.41(-0.42-1.01)$ & $<0.001$ & $<0.001$ & 0.18 \\
\hline LLD percentile & $90(75-95)$ & $75(50-90)$ & $50(25-75)$ & $<0.001$ & $<0.001$ & 0.65 \\
\hline Triglyceride $(\mathrm{mg} / \mathrm{dl})$ & $125(88-149)$ & $123(92-158)$ & $82(63-117)$ & $<0.001$ & $<0.001$ & 0.46 \\
\hline Total cholesterol (mg/dl) & $167( \pm 29)$ & $164( \pm 27)$ & $141( \pm 25)$ & $<0.001$ & $<0.001$ & 0.84 \\
\hline $\operatorname{ALT}(U / L)$ & $19(16-26)$ & $19(15-23)$ & $17(12-21)$ & $<0.001$ & $<0.001$ & 0.99 \\
\hline Fasting insulin $(\mu \mathrm{U} / \mathrm{ml})$ & $17.6(13.2-22.88)$ & $17.9(12.6-23.4)$ & $9.24(7.3-11.6)$ & $<0.001$ & $<0.001$ & 0.96 \\
\hline HOMA-IR & $3.57(2.92-5.10)$ & $3.56(2.47-4.60)$ & $1.94(1.47-2.60)$ & $<0.001$ & $<0.001$ & 0.98 \\
\hline LVMI $\left(\mathrm{g} / \mathrm{m}^{2.7}\right)$ & $40.4(35.8-46.1)$ & $39.6(34.6-45.6)$ & $29.7(25.2-33.6)$ & $<0.001$ & $<0.001$ & 0.92 \\
\hline
\end{tabular}

*The data are expressed as the mean \pm SD or median (25\%-75\%) where appropriate; BMI-SDS: Body Mass Index - Standard Deviation Score; LLD: Liver Longitudinal Dimension Percentile; ALT: Alanine Aminotransferase; HOMA-IR: Homeostasis Model Assessment of Insulin Resistance; NAFLD: Non-Alcoholic Fatty Liver Disease;

LVMI: Left Ventricle Mass Index; LVH: Left Ventricle Hypertrophy; NAFLD: Non-Alcoholic Fatty Liver Disease

$p 1$ : obese children with NAFLD vs. control; $p 2$ : obese children without NAFLD vs. control; $p 3$ : obese children with NAFLD vs. obese without NAFLD

Table 2: Obesity related risk factors in obese children with NAFLD, obese children without NAFLD, and control subjects*.

percentile as independent variables. Logarithmic transformed data for LVM and LVMI were used because of their non-normal distributions. Multiple linear regression analysis was performed with LVMI as a dependent variable, NAFLD as a main predictor variable, and BMI-SDS as a covariate (model I). In model II, LVMI was the dependent variable, NAFLD was the main predictor variable, and BMI-SDS, SBP, and DBP were covariates. $\mathrm{P}$-values $<0.05$ were considered statistically significant.

\section{Results}

Of the participants, six were excluded because they tested positive for HBsAg $(n=5)$ or anti-HCV $(n=1)$. The study included 98 obese children and 48 non-obese control children. Of the obese subjects, 43 had NAFLD and 55 did not. The physical characteristics, laboratory results, LLD, LVM, and LVMI of the obese children and controls are summarized in Table 1. Compared with the controls, the obese children had significant differences in several clinical risk factors, including body weight, BMI, BMI-SDS, SBP, DBP, waist circumference, hip circumference, and waist/hip ratio (all $p<0.001$ ). Nineteen $(19.4 \%$ ) of the 98 obese children had systolic or diastolic hypertension; none of the control subjects did. Insulin resistance was seen in $66(67 \%)$ of the obese children and only two (4\%) of the controls. The median LLD percentile in obese children and control subjects was the 82.5 (5-95) and $50(10-95)$ percentile, respectively $(p<0.001)$. Forty-three $(44 \%)$ of the obese children had NAFLD, while only one $(2 \%)$ control subject did. The median LVMI in obese and control subjects was 40.1 (26.4$68.2)$ and $29.7(15.6-42.0) \mathrm{g} / \mathrm{m}^{2.7}$, respectively $(p<0.001)$. Comparisons of obesity-related risk factors between obese children with and without NAFLD and control subjects are presented in Table 2. All obesityrelated risk factors differed significantly between the controls and obese children with or without NAFLD (all $p<0.001$; Table 2). However, the same variables did not differ between obese children with and without NAFLD (Figure 1). LVH was present in 5 of 48 (10.4\%) control subjects and 30 of 98 (30.6\%) obese subjects. When obese children were grouped according to whether or not they had hypertension, $\mathrm{LVH}$ was present in 24 of 79 (30.3\%) obese subjects without hypertension and 6 of $19(36.8 \%)$ obese subjects with hypertension. The frequency of LVH differed significantly between the obese children and controls 
Citation: Selver B, Simsek E,Kocabas U, Dallar Y (2012) Non-Alcoholic Fatty Liver Disease and the Left Ventricle Mass Index in Obese Children. J Metabolic Synd 1:110. doi:10.4172/2167-0943.1000110

Page 4 of 7

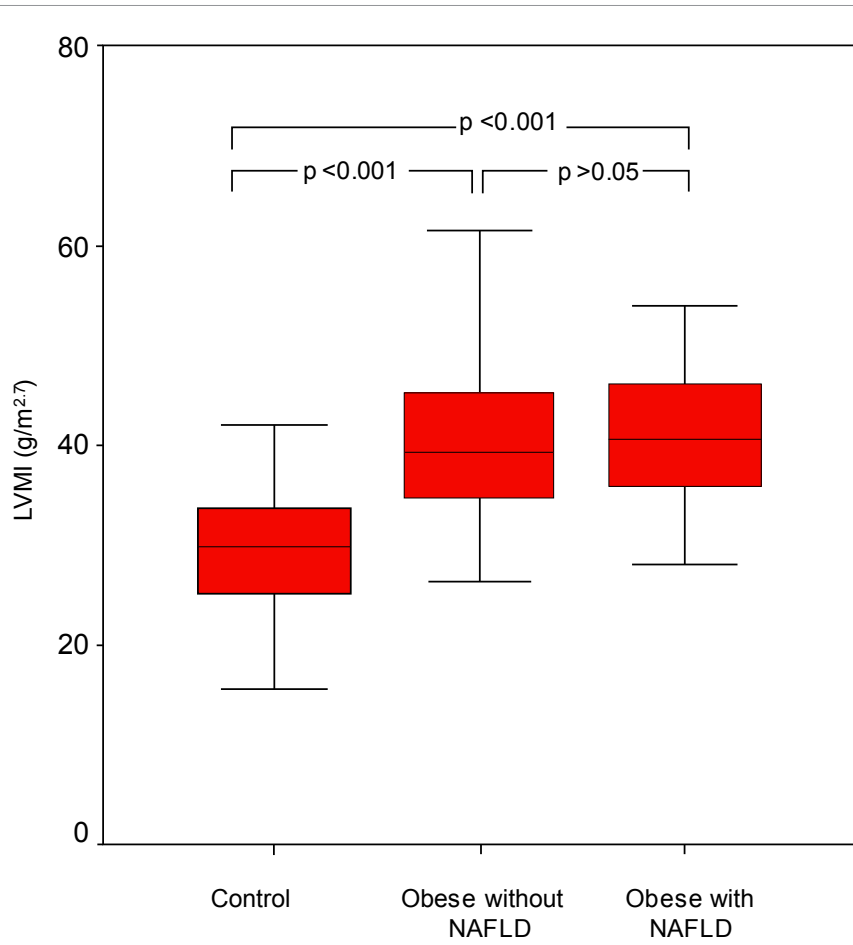

Figure 1: Adjusted pair-wise comparison of the left ventricle mass index (LVMI) of obese children with non-alcoholic fatty liver disease (NAFLD), obese children without NAFLD, and control subjects.

\begin{tabular}{|l|c|c|}
\hline & \multicolumn{2}{|c|}{ BMI-SDS } \\
\hline Glucose & $r$ & $p$ \\
\hline Insulin & -0.075 & 0.369 \\
\hline HOMA-IR & 0.470 & $<0.001$ \\
\hline Triglyceride & 0.436 & $<0.001$ \\
\hline Total cholesterol & 0.362 & $<0.001$ \\
\hline AST & 0.446 & $<0.001$ \\
\hline ALT & 0.033 & 0.694 \\
\hline
\end{tabular}

HOMA-IR: Homeostasis Model Assessment of Insulin Resistance;

AST: Aspartate Aminotransferase; ALT: Alanine Aminotransferase

Table 3: Univariate Spearman's correlation coefficients between the study variables and body mass index-standart deviation scores (BMI-SDS).

\begin{tabular}{|l|c|c|c|c|}
\hline & \multicolumn{2}{|c|}{ LVM } & \multicolumn{2}{c|}{ LVMI } \\
\hline Age & $r$ & $p$ & $r$ & $p$ \\
\hline BMI-SDS & 0.526 & $<0.001$ & 0.094 & 0.259 \\
\hline HOMA-IR & 0.561 & $<0.001$ & 0.675 & $<0.001$ \\
\hline AST & 0.490 & $<0.001$ & 0.250 & 0.002 \\
\hline ALT & 0.221 & 0.007 & 0.007 & 0.935 \\
\hline LLD percentile & 0.060 & 0.473 & 0.155 & 0.062 \\
\hline
\end{tabular}

BMI-SDS: Body Mass Index-Standart Deviation Scores; HOMA-IR: Homeostasis Model Assessment of Insulin Resistance; AST: Aspartate Aminotransferase; ALT: Alanine Aminotransferase; LLD: Liver Longitudinal Dimension; LVM: Left Ventricle Mass

Table 4: Univariate Spearman's correlation coefficients between the study variables and left ventricle mass (LVM) and LVMI index.

$(p<0.001)$, whereas no significant difference was observed between the obese children with and without hypertension. The serum ALT differed significantly between obese children with or without NAFLD and control subjects (all $p<0.001$ ). Eight $(18.6 \%)$ of 43 obese children with NAFLD had an abnormal ALT.

\begin{tabular}{|l|l|c|c|c|c|}
\hline Models & & $\beta$ & $\begin{array}{c}95 \% \text { Confidence } \\
\text { Interval } \\
\text { Lower Limit }\end{array}$ & Upper Limit & \\
\hline Model I & (Constant) & 3.378 & 3.316 & 3.440 & $<0.001$ \\
\hline & NAFLD & 0.042 & -0.044 & 0.128 & 0.333 \\
\hline & Obesity & 0.308 & 0.225 & 0.392 & $<0.001$ \\
\hline Model II & (Constant) & 3.357 & 3.282 & 3.431 & $<0.001$ \\
\hline & NAFLD & 0.040 & -0.046 & 0.126 & 0.358 \\
\hline & Obesity & 0.305 & 0.217 & 0.392 & $<0.001$ \\
\hline & SBP Percentile & 0.007 & -0.021 & 0.035 & 0.627 \\
\hline & DBP Percentile & 0.004 & -0.002 & 0.010 & 0.218 \\
\hline
\end{tabular}

NAFLD: Non-Alcoholic Fatty Liver Disease; SBP: Systolic Blood Pressure; DBP Diastolic Blood Pressure

Table 5: Multiple linear regression analysis for predictors of left-ventricle mass in$\operatorname{dex}($ LVMI)

In the univariate Spearman's correlation analysis, BMI-SDS was directly related to the fasting insulin level, HOMA-IR, TG, TC, and ALT levels (Table 3), whereas, LVMI was positively correlated with BMI-SDS, HOMA-IR, and LLD percentile (Table 4). In the multiple linear regression analysis, LVMI $(\beta=0.119 ; p<0.001)$ and NAFLD $(\beta=0.213 ; p<0.001)$ remained independent correlates of BMI-SDS. The Spearman's correlation analysis indicated no significant correlation between blood pressure and LVMI. In multiple linear regression models with NAFLD, BMI-SDS, SBP, and DBP as covariates, NAFLD was a significant predictor of LVMI $(\beta=3.4, p<0.001)$. When BMI-SDS was added to the model, NAFLD as a predictor of LVMI was strongly attenuated and no longer statistically significant $(\beta=0.4)$. BMI-SDS remained a significant predictor of LVMI $(\beta=0.3, p<0.001)$. When SBP and DBP were added to the model, the NAFLD association was also attenuated $(\beta=0.4)$. BMI-SDS remained a significant predictor of LVMI $(\beta=0.3, p<0.001)$. SBP and DBP were not significant predictors of LVMI $(\beta=0.007)$ (Table 5).

\section{Discussion}

In addition to the current rise in obesity, NAFLD is becoming responsible for a large percentage of liver disease in children [1]. The actual prevalence of NAFLD remains unknown largely because of the lack of population-based studies and reliable noninvasive screening tools. The reported frequency of NAFLD is $20 \%$ of obese children and adolescents from the US [22], 44\% from Italy [23], and 74\% from China [24]. In the present study, the frequency of NAFLD was $44 \%$ in obese subjects.

NAFLD encompasses a spectrum of diseases, from asymptomatic steatohepatitis to cirrhosis. The pathogenesis of NAFLD has remained poorly understood since the earliest description of the disease. Studies have reported the pathophysiology of NAFLD in obese patients in detail $[1,25,26]$. Insulin resistance and oxidative stress have critical roles in NAFLD pathogenesis. The molecular basis of the association between insulin resistance and hepatic steatosis is unclear. In this study, fasting insulin level, HOMA-IR ALT (as a parameter of insulin resistance), TG, TC, and ALT did not differ between obese children with and without NAFLD. In multiple regression analyses, insulin resistance itself was not an independent predictor of NAFLD, whereas the severity of obesity (as BMI-SDS) showed a significant correlation with NAFLD. In contrast, some studies have reported that insulin resistance is associated with the development of NAFLD in obese subjects $[27,28]$. Taken together, previous results and our current findings suggest that the molecular pathogenesis of insulin resistance is multifactorial. Mildly to moderately elevated serum ALT or AST levels are the most common and often the only laboratory abnormalities in NAFLD. In previous studies, the frequency of an elevated ALT level was between 10 
and 30\% in subjects with NAFLD [29,30]. In our study, the ALT levels differed significantly between obese children and controls, whereas no significant difference was observed between obese children with and without NAFLD. Only 8 of $43(18.6 \%)$ obese children with NAFLD had abnormal ALT levels. A large portion of obese children may be missed at screening based only on serum aminotransferases. Fishbein et al. [31] reported that enzymatic abnormalities in fatty livers occur only in severe cases. Furthermore, Mofrad et al. [32] reported that the entire spectrum of NAFLD could be seen in subjects with normal ALT values. An ultrasound examination is preferred for identifying a fatty liver due to its noninvasiveness, availability, and high sensitivity and specificity. Joseph et al. [33] reported a sensitivity of $89 \%$ and specificity of $93 \%$ for detecting steatosis in the liver. Furthermore, we found that no subject with a normal liver ultrasound had an elevated ALT, and ultrasound revealed liver steatosis in all patients with elevated ALT levels. These findings suggest that an elevated ALT, higher BMI-SDS, and echogenic liver parenchyma, revealed by ultrasonography, are predictors of NAFLD.

The other obesity-related cardiovascular risk factor includes an increased LVMI or LVH. A number of studies have examined the relationship between LVM, BP, and obesity (as BMI or BMI-SDS) with conflicting results. A high $\mathrm{BP}$ (i.e., $\mathrm{BP}>95^{\text {th }}$ percentile) of between 18 and $35 \%$ has been reported in overweight and obese children [34,35]. In this study, 19 of 98 (19.4\%) obese children were hypertensive. The frequency of LVH differed significantly between the obese children and controls, whereas no significant difference was observed between the obese children with and without hypertension. In a multiple linear regression analysis of the obesity-related risk factors (BMI-SDS, glucose, fasting insulin levels, HOMA-IR, TC, and TGs) that may be responsible for the pathogenesis of increased LVMI, BMI-SDS was the only significant predictor of increased LVMI. Furthermore, in a multiple linear regression analysis, BP and NAFLD were not significant predictors of LVMI. Our findings are supported by previous studies [2,36-39]. The Bogalusa Heart Study showed a strong association between LVM in childhood and the degree of obesity, but childhood BMI was the only independent predictor for adult LVMI [2]. Dhuper et al. [40] reported that obesity is a major predictor of concentric remodeling (CR) of the left ventricle, and the prevalence of $\mathrm{CR}$ of the left ventricle was similar in both the hypertensive and normotensive obese groups. Daniels et al. [41] showed a strong association between BMI and increased LVMI, independent of SBP. Finally, adult studies also reported that LVM is increased in normotensive obese adults, and that it is more closely associated with BMI and insulin resistance rather than BP $[42,43]$. Obesity affects cardiac muscle through multiple mechanisms. Factors other than BP, such as increased sympathetic activity, insulin resistance, vascular remodeling, neurohumoral modulation, volume overload, endothelial dysfunction, oxidative stress, and inflammation, which were not evaluated in the present study, may play a role in the pathogenesis of increasing LVMI in obese subjects. In contrast, some studies have shown that LVMI is positively associated with elevated BP [44-46]. Sorof et al. [47] found that LVMI is strongly correlated with 24-hour ambulatory measurements of the SBP index, whereas LVMI did not correlate with casual BP. Maggio et al. [48] reported a similar observation. In contrast, de Simone et al. [46] reported that the risk of LVH was significantly higher in children with a high casual BP as compared with children with normal BP, independent of the effects of obesity. These contradictory findings may be explained by different BP measurement methods and masked hypertension, which is defined as elevated ambulatory BP measurement without concomitant elevation of casual BP measurement.

Although NAFLD and increased LVMI or LVH are two important obesity-related covariates, this is the first study to identify a relationship between NAFLD and LVMI in obese children. Components of Metabolic Syndrome (MS, i.e., hypertension, hyperinsulinism, dyslipidemia, impaired glucose tolerance, and obesity) are strongly associated with NAFLD [49,50]. Children with NAFLD often have multiple cardiovascular risk factors, including abnormal waist circumference, dyslipidemia, hypertension, insulin resistance [51], and increased carotid intima media thickness [52]. In a case control study comparing 150 pediatric patients with biopsy-proven NAFLD versus 150 overweight controls, children with MS had a five-fold increased risk of NAFLD [51]. Considering these studies, it can be concluded that NAFLD represents a liver manifestation of MS. It is well known that a major risk factor for mortality from MS is cardiovascular disease. Studies have shown that mortality among patients with NAFLD is higher than that in the general population, mainly due to concomitant cardiovascular diseases [53-56]. The natural history of NAFLD in the pediatric population is not clearly understood due to a lack of prospective studies evaluating children over time $[57,58]$. In our study, LVMI was significantly higher in obese children with or without NAFLD as compared with non-obese children. However, LVMI was not significantly different between obese subjects with or without NAFLD. Furthermore, the multiple linear regression analysis revealed that NAFLD was not a significant predictor for LVMI and that BMI-SDS was the only main predictor of LVMI. The findings of this study highlight that there is no relationship between NAFLD and LVMI. This finding may be explained by the different pathophysiology of NAFLD and increased LVMI in obese children. The higher mortality rate secondary to cardiovascular diseases in NAFLD may be explained because NAFLD is a significant predictor of liver damage in MS.

Our study had two limitations. The current study was crosssectional in design; therefore, temporal associations between obesity, BP elevation, LVMI, and NAFLD could not been evaluated. We used the method of casual BP measurement to screen for hypertension. Casual BP may not reflect the most comprehensive view of BP and may miss a diagnosis of hypertension.

In conclusion, this study has demonstrated that LVH and NAFLD are two important and independent obesity-related covariates in obese children. Obese children with or without hypertension have a higher frequency of LVH compared to non-obese control subjects. In addition, LVMI may be influenced by fat mass rather than casual BP. The 24-hour ambulatory BP measurement may be a potential tool to improve risk stratification in obesity studies. NAFLD and casual BP measurements are not predictors of LVMI in obese children. Collectively, NAFLD is an obesity-related risk factor for chronic liver disease and is expected to become one of the most common causes of end-stage liver disease in children.

\section{References}

1. Pacifico L, Nobili V, Anania C, Verdecchia P, Chiesa C (2011) Pediatric nonalcoholic fatty liver disease, metabolic syndrome and cardiovascular risk. World J Gastroenterol 17: 3082-3091.

2. Li X, Li S, Ulusoy E, Chen W, Srinivasan SR, et al. (2004) Childhood adiposity as a predictor of cardiac mass in adulthood: the Bogalusa Heart Study. Circulation 110: 3488-3492.

3. Serdual MK, Ivery D, Coates RJ, Freedman DS, Williamson DF, et al. (1993) Do obese children become obese adults? A review of the literature. Prev Med 22 167-177.

4. Chan DF, Li AM, Chu WC, Chan MH, Wong EM, et al. (2004) Hepatic steatosis in obese Chinese children. Int J Obes Relat Metab Disord 28: 1257-1263.

5. Tominaga K, Kurata JH, Chen YK, Fujimoto E, Miyagawa S, et al. (1995) Prevalence of fatty liver in Japanese children and relationship to obesity. An epidemiological ultrasonographic survey. Dig Dis Sci 40: 2002-2009. 
6. Franzese A, Vajro P, Argenziano A, Puzziello A, lannucci MP, et al. (1997) Liver involvement in obese children. Ultrasonography and liver enzyme levels at diagnosis and during follow-up in an Italian population. Dig Dis Sci 42: 14281432

7. Hanevold C, Waller J, Daniels S, Portman R, Sorof J, et al. (2004) The effects of obesity, gender, and ethnic group on left ventricular hypertrophy and geometry in hypertensive children: a collaborative study of the International Pediatric Hypertension Association. Pediatrics 113: 328-333.

8. Galvan AQ, Galetta F, Natali A, Muscelli E, Sironi AM, et al. (2000) Insulin resistance and hyperinsulinemia: No independent relation to left ventricular mass in humans. Circulation 102: 2233-2238.

9. Cole TJ, Bellizzi MC, Flegal KM, Dietz WH (2000) Establishing a standard definition for child overweight and obesity worldwide: international survey. BM 320: $1240-1243$.

10. Bundak R, Furman A, Gunoz H, Darendeliler F, Bas F, et al. (2006) Body mass index references for Turkish children. Acta Paediatr 95: 194-198.

11. Soergel M, Kirschstein M, Busch C, Danne T, Gellermann J, et al. (1997) Oscillometric twenty-four-hour ambulatory blood pressure values in healthy children and adolescents: a multicenter trial including 1141 subjects. J Pediatr 130: 178-184.

12. Tümer N, Yalçinkaya F, Ince E, Ekim M, Köse K, et al. (1999) Blood pressure nomograms for children and adolescents in Turkey. Pediatr Nephrol 13: 438443.

13. Matthews DR, Hosker JP, Rudenski AS, Naylor BA, Treacher DF, et al. (1985) Homeostasis model assessment: insulin resistance and beta-cell function from fasting plasma glucose and insulin concentrations in man. Diabetologia 28 $412-419$

14. Keskin M, Kurtoglu S, Kendirci M, Atabek ME, Yazici C (2005) Homeostasis model assessment is more reliable than the fasting glucose/insulin ratio and quantitative insulin sensitivity check index for assessing insulin resistance among obese children and adolescents. Pediatrics 115: e500-e503.

15. (1992) American Academy of Pediatrics. National Cholesterol Education Program: Report of the Expert Panel on Blood Cholesterol Levels in Children and Adolescents. Pediatrics 89: 525-584.

16. Needleman L, Kurtz AB, Rifkin MD, Cooper HS, Pasto ME, et al. (1986) Sonography of diffuse benign liver disease: accuracy of pattern recognition and grading. AJR Am J Roentgenol 146: 1011-1015.

17. Schiller NB, Shah PM, Crawford M, DeMaria A, Devereux R, et al. (1989) Recommendations for quantitation of the left ventricle by two-dimensional echocardiography. American Society of Echocardiography Committee on Standards, Subcommittee on Quantitation of Two-Dimensional Echocardiograms. J Am Soc Echocardiogr 2: 358-367.

18. Devereux RB, Alonso DR, Lutas EM, Gottlieb GJ, Campo E, et al. (1986) Echocardiographic assessment of left ventricular hypertrophy: comparison to necropsy findings. Am J Cardiol 57: 450-458.

19. de Simone G, Daniels SR, Devereux RB, Meyer RA, Roman MJ, et al. (1992) Left ventricular mass and body size in normotensive children and adults: assessment of allometric relations and impact of overweight. J Am Coll Cardiol 20: $1251-1260$

20. de Simone G, Devereux RB, Daniels SR, Koren MJ, Meyer RA, et al. (1995) Effect of growth on variability of left ventricular mass: assessment of allometric signals in adults and children and their capacity to predict cardiovascular risk. J Am Coll Cardiol 25: 1056-1062.

21. Daniels SR (1999) Hypertension-induced cardiac damage in children and adolescents. Blood Press Monit 4: 165-170.

22. Strauss RS, Barlow SE, Dietz WH (2000) Prevalence of abnormal serum aminotransferase values in overweight and obese adolescents. J Pediatr 136: 727-733.

23. Sartorio A, Del Col A, Agosti F, Mazzilli G, Bellentani S, et al. (2007) Predictors of non-alcoholic fatty liver disease in obese children. Eur J Clin Nutr 61: 877883

24. Chan DF, Li AM, Chu WC, Chan MH, Wong EM, et al. (2004) Hepatic steatosis in obese Chinese children. Int J Obes Relat Metab Disord 28: 1257-1263.

25. Alisi A, Manco M, Vania A, Nobili V (2009) Pediatric nonalcoholic fatty liver disease in 2009. J Pediatr 155: 469-474.

26. Feldstein AE, Charatcharoenwitthaya P, Treeprasertsuk S, Benson JT, Enders
FB, et al. (2009) The natural history of non-alcoholic fatty liver disease in children: a follow-up study for up to 20 years. Gut 58: 1538-1544.

27. Feldstein AE (2010) Novel insights into the pathophysiology of nonalcoholic fatty liver disease. Semin Liver Dis 30: 391-401.

28. Fabbrini E, Sullivan S, Klein S (2010) Obesity and nonalcoholic fatty liver disease: biochemical, metabolic, and clinical implications. Hepatology 51: 679689

29. Tazawa Y, Noguchi H, Nishinomiya F, Takada G (1997) Serum alanine aminotransferase activity in obese children. Acta Paediatr 86: 238-241.

30. Vajro P, Fontanella A, Perna C, Orso G, Tedesco M, et al. (1994) Persistent hyperaminotransferasemia resolving after weight reduction in obese children. $J$ Pediatr 25: 239-241.

31. Fishbein MH, Miner M, Mogren C, Chalekson J (2003) The spectrum of fatty liver in obese children and the relationship of serum aminotransferases to severity of steatosis. J Pediatr Gastroenterol Nutr 36: 54-61.

32. Mofrad P, Contos MJ, Haque M, Sargeant C, Fisher RA, et al. (2003) Clinica and histologic spectrum of nonalcoholic fatty liver disease associated with normal ALT values. Hepatology 37: 1286-1292.

33. Joseph AE, Saverymuttu SH, al-Sam S, Cook MG, Maxwell JD (1991) Comparison of liver histology with ultrasonography in assessing diffuse parenchymal liver disease. Clin Radiol 43: 26-31.

34. Raj M, Sundaram KR, Paul M, Deepa AS, Kumar RK (2007) Obesity in Indian children: time trends and relationship with hypertension. Natl Med J India 20 288-293.

35. l'Allemand D, Wiegand S, Reinehr T, Müller J, Wabitsch M, et al. (2008) Cardiovascular risk in 26,008 European overweight children as established by a multicenter database. Obesity (Silver Spring) 16: 1672-1679.

36. Friberg P, Allansdotter-Johnsson A, Ambring A, Ahl R, Arheden H, et al. (2004 Increased left ventricular mass in obese adolescents. Eur Heart J 25: 987-992.

37. Kinik ST, Varan B, Yildirim SV, Tokel K (2006) The effect of obesity on echocardiographic and metabolic parameters in childhood. J Pediatr Endocrino Metab 19: 1007-1014.

38. Van Putte-Katier N, Rooman RP, Haas L, Verhulst SL, Desager KN, et al (2008) Early cardiac abnormalities in obese children: importance of obesity per se versus associated cardiovascular risk factors. Pediatr Res 64: 205-209.

39. Chinali M, de Simone G, Roman MJ, Lee ET, Best LG, et al. (2006) Impact of obesity on cardiac geometry and function in a population of adolescents: the Strong Heart Study. J Am Coll Cardiol 47: 2267-2273.

40. Dhuper S, Abdullah RA, Weichbrod L, Mahdi E, Cohen HW (2011) Association of obesity and hypertension with left ventricular geometry and function in children and adolescents. Obesity (Silver Spring) 19: 128-133.

41. Daniels SR, Witt SA Glascock B, Khoury PR, Kimball TR (2002) Left atrial size in children with hypertension: the influence of obesity, blood pressure, and left ventricular mass. J Pediatr 141: 186-190.

42. Wong CY, O'Moore-Sullivan T, Leano R, Byrne N, Beller E, et al. (2004) Alterations of left ventricular myocardial characteristics associated with obesity. Circulation 110: 3081-3087

43. lacobellis G, Ribaudo MC, Zappaterreno A, Vecci E, Tiberti C, et al. (2003) Relationship of insulin sensitivity and left ventricular mass in uncomplicated obesity. Obes Res 11: 518-524

44. Daniels SR, Kimball TR, Morrison JA, Khoury P, Witt S, et al. (1995) Effect of lean body mass, fat mass, blood pressure, and sexual maturation on left ventricular mass in children and adolescents. Statistical, biological, and clinical significance. Circulation 92: 3249-3254.

45. Malcolm DD, Burns TL, Mahoney LT, Lauer RM (1993) Factors affecting left ventricular mass in childhood: The Muscatine Study. Pediatrics 92: 703-709.

46. de Simone G, Mureddu GF, Greco R, Scalfi L, Del Puente AE, et al. (1997) Relations of left ventricular geometry and function to body composition in children with high casual blood pressure. Hypertension 30: 377-382.

47. Sorof JM, Cardwell G, Franco K, Portman RJ (2002) Ambulatory blood pressure and left ventricular mass index in hypertensive children. Hypertension 39: 903 908

48. Maggio AB, Aggoun Y, Marchand LM, Martin XE, Herrmann F, et al. (2008) Associations among obesity, blood pressure, and left ventricular mass. J Pediatr 152: 489-493. 
Citation: Selver B, Simsek E, Kocabas U, Dallar Y (2012) Non-Alcoholic Fatty Liver Disease and the Left Ventricle Mass Index in Obese Children. J Metabolic Synd 1:110. doi:10.4172/2167-0943.1000110

Page 7 of 7

49. Loria P, Lonardo A, Carulli L, Verrone AM, Ricchi M, et al. (2005) Review article: the metabolic syndrome and non-alcoholic fatty liver disease. Aliment Pharmacol Ther 22: 31-36.

50. Mencin AA, Lavine JE (2011) Nonalcoholic fatty liver disease in children. Curr Opin Clin Nutr Metab Care 14: 151-157.

51. Schwimmer JB, Pardee PE, Lavine JE, Blumkin AK, Cook S (2008) Cardiovascular risk factors and the metabolic syndrome in pediatric nonalcoholic fatty liver disease. Circulation 118: 277-283.

52. Demircioğlu F, Koçyiğit A, Arslan N, Cakmakçi H, Hizli S, et al. (2008) Intimamedia thickness of carotid artery and susceptibility to atherosclerosis in obese children with nonalcoholic fatty liver disease. J Pediatr Gastroenterol Nutr 47: 68-75

53. Adams LA, Lymp JF, St Sauver J, Sanderson SO, Lindor KD, et al. (2005) The natural history of nonalcoholic fatty liver disease: a population-based cohort study. Gastroenterology 129: 113-121.
54. Ekstedt M, Franzén LE, Mathiesen UL, Thorelius L, Holmqvist M, et al. (2006) Long-term follow-up of patients with NAFLD and elevated liver enzymes. Hepatology 44: 865-873.

55. Rafiq N, Bai C, Fang Y, Srishord M, McCullough A, et al. (2009) Long-term follow-up of patients with nonalcoholic fatty liver. Clin Gastroenterol Hepatol 7: 234-238.

56. Söderberg C, Stål P, Askling J, Glaumann H, Lindberg G, et al. (2010) Decreased survival of subjects with elevated liver function tests during a 28year follow-up. Hepatology 51: 595-602.

57. Loomba R, Sirlin CB, Schwimmer JB, Lavine JE (2009) Advances in pediatric nonalcoholic fatty liver disease. Hepatology 50: 1282-1293.

58. Argo CK, Northup PG, Al-Osaimi AM, Caldwell SH (2009) Systematic review of risk factors for fibrosis progression in non-alcoholic steatohepatitis. J Hepatol 51: $371-379$ 